\title{
Case Report \\ The Clinical Cases of Geleophysic Dysplasia: One Gene, Different Phenotypes
}

\author{
Evgenia Globa $\mathbb{D}^{1},{ }^{1}$ Nataliya Zelinska, ${ }^{1}$ and Andrew Dauber ${ }^{2}$ \\ ${ }^{1}$ Ukrainian Research Center of Endocrine Surgery, Pediatric Endocrinology Department, Kyiv, Ukraine \\ ${ }^{2}$ Division of Endocrinology, Cincinnati Center for Growth Disorders, Cincinnati Children's Hospital Medical Center, USA \\ Correspondence should be addressed to Evgenia Globa; ie.globa@i.ua
}

Received 27 February 2018; Revised 25 April 2018; Accepted 28 May 2018; Published 3 July 2018

Academic Editor: Osamu Isozaki

Copyright (c) 2018 Evgenia Globa et al. This is an open access article distributed under the Creative Commons Attribution License, which permits unrestricted use, distribution, and reproduction in any medium, provided the original work is properly cited.

\begin{abstract}
Background. Geleophysic dysplasia is a rare multisystem disorder that principally affects the bones, joints, heart, and skin. This condition is inherited either in an autosomal dominant pattern due to FBN1 mutations or in an autosomal recessive pattern due to ADAMTSL2 mutations. Two patients with unaffected parents from unrelated families presented to their endocrinologist with severe short stature, resistant to growth hormone treatment. Routine endocrine tests did not reveal an underlying etiology. Exome sequencing was performed in each family. Our two patients, harboring de novo heterozygous FBN1 mutations p.Tyr1696Asp and p.Cys1748Ser, had common clinical symptoms such as severe short stature, characteristic facial features, short hands and feet, and limitation of joint movement. However, one patient had severe cardiac involvement whereas the other patient had tracheal stenosis requiring tracheostomy placement. Conclusions. Patients with severe dwarfism, skeletal anomalies, and other specific syndromic features (e.g., tracheal stenosis and cardiac valvulopathy) should undergo genetic testing to exclude acromelic dysplasia syndromes.
\end{abstract}

\section{Introduction}

Geleophysic dysplasia (GD) (MIM \#231050, 617809, and 614185), acromicric dysplasia (AD) (MIM \#102370), and Weill-Marchesani syndrome (WMS) (MIM \#614819, 608328, 613195 , and 277600) are rare disorders with overlapping characteristics including short stature, short hands and feet, progressive joint limitations and contractures, skin thickening, distinctive facial features, and abnormal skeletal morphology [1-7]. AD usually has a less severe outcome due to the absence of progressive cardiac valvular thickening, whereas a distinctive feature in WMS patients is an abnormality of the lens of the eye [1].

GD is characterized by short stature, prominent abnormalities in hands and feet, bones, and joints, and progressive cardiac, respiratory, and lung pathology with a characteristic facial appearance (described as gelios = happy, and physis $=$ nature) [1-4]. The three genes known to be associated with geleophysic dysplasia are ADAMTSL2 (geleophysic dysplasia 1), FBN1 (geleophysic dysplasia 2), and the recently discovered latent transforming growth factor beta (TGF $\beta$ )binding protein-3 (LTBP3) [5-7]. FBN1 encodes fibrillin-1 and ADAMTSL2 (a disintegrin and metalloproteinase with thrombospondin repeats-like 2) encodes a glycoprotein of unknown function. All FBN1 pathogenic variants identified to date in GD are clustered in the same region encoding the TGF $\beta$-binding protein-like domain 5 (TB5) domain of FBN1 $[3,6]$.

Mutations in FBN1 are also associated with a large spectrum of diseases including Marfan syndrome, WeillMarchesani syndrome, Stiff skin syndrome, MASS syndrome, and Marfan lipodystrophy syndrome [8-10]. The mechanism by which changes in this gene contribute to both tall and short stature is still unclear [3].

Transmission of GD is variable and corresponds to an autosomal recessive model in the cases with ADAMTSL2 gene mutations and an autosomal dominant model in the cases with FBN1 and $L T B P 3$ mutations $[1,7]$. The true prevalence of GD in the world is unknown; however approximately 55 affected individuals have been reported in 2009 [1] giving 
a prevalence $<1 / 1000000$. Symptoms of GD can vary widely from person to person and thus the diagnosis of this rare condition can be quite complicated. Many patients may remain un- or misdiagnosed.

In the present study, we report two patients from unrelated families who had highly variable clinical presentations of GD but their common feature was severe short stature with resistance to growth hormone $(\mathrm{GH})$ treatment. Nevertheless, exome sequencing identified the presence of de novo heterozygous FBN1 variants in both patients. This study was approved by the Institutional Review Board at Cincinnati Children's Hospital Medical Center (Protocol \#2014-5919). Written informed consent was obtained from both patients' parents.

\section{Case Presentation}

Patient 1 (P1) is a Ukrainian girl who was born as the second child of nonconsanguineous white parents following an uneventful pregnancy and spontaneous term delivery. She had been born at a gestational age of 39 weeks with a normal birth weight $\left(3100 \mathrm{~g}, 15^{\text {th }}-50^{\text {th }}\right.$ percentile) and birth length $\left(50 \mathrm{~cm}, 50^{\text {th }}-85^{\text {th }}\right.$ percentile). Progressive postnatal growth delay developed since 1 year of age. Familial stature was well within the normal range with a maternal height of 154 $\mathrm{cm}(-1.3 \mathrm{SD})$, paternal height of $175 \mathrm{~cm}(+0.04 \mathrm{SD})$, and a brother's final height of $169 \mathrm{~cm}(-0.8 \mathrm{SD}$ ) (Figure 1(a)). The patient was first examined by an endocrinologist at the age of 2.5 years. Her height was $72 \mathrm{~cm}(-4.5 \mathrm{SD})$ and weight was $7.9 \mathrm{~kg}\left(<5^{\text {th }}\right.$ percentile). Physical examination revealed additional dysmorphic features and other physical abnormalities including a broad nasal bridge, a bulbous nose, elongation of the eye lashes, contractures of the elbow joints and wrists, and small hands and fingers (Figure 1(b)). Routine biochemical analysis demonstrated normal hematology, chemistry, and thyroid hormone function. IGF-1 was $51.7 \mathrm{ng} / \mathrm{ml}$ (between the 10 th and 50th percentile).

Further investigation was done at the age of 6 years and revealed a normal clonidine-stimulated $\mathrm{GH}$ peak of $25 \mathrm{ng} / \mathrm{ml}$. Baseline IGF-1 was $74.4 \mathrm{ng} / \mathrm{ml}$ (between the 10th and 50th percentile). IGF-1 generation test showed no response to $\mathrm{GH}$ stimulation (after three days of $\mathrm{GH}$ administration at a dose $0.03 \mathrm{mg} / \mathrm{kg} /$ day IGF-1 remained low at $55.2 \mathrm{ng} / \mathrm{ml}$ ). Karyotype was that of a normal female (46 XX). Her bone age at her calendar age of 6 years was 2 years (as assessed by the Greulich and Pyle method), and cone-shaped epiphyses and Madelung deformity were noted (Figures 1(b) and 1(c)). Skeletal Xray of the lower extremities showed lateral positioning of the femoral heads and varus deformity of the knee joints (Figure 1(d)). A brain MRI revealed a hypoplastic pituitary and sella turcica. She was prescribed empiric treatment with recombinant $\mathrm{GH}(\mathrm{rGH})$ at a dose $0.03 \mathrm{mg} / \mathrm{kg} /$ day which was ineffective. Over a period of 4 months, the child grew by only $0.8 \mathrm{~cm}$. Thereafter, $\mathrm{rGH}$ therapy was discontinued. Further observation showed progressive growth retardation (Figures 1(e) and 1(f)). At 8 years of age, her height was $80.5 \mathrm{~cm}(-8 \mathrm{SD})$ and weight was $11 \mathrm{~kg}\left(<5^{\text {th }}\right.$ percentile). At 7 years of age, echocardiography showed minimal aortic, mitral, and pulmonary stenosis. However, two years later, a repeat echocardiogram showed worsening cardiac disease with progression of the mild aortic, mitral, and pulmonary stenosis and new findings of pulmonary hypertension and left ventricular hypertrophy. The patient also suffers from carpal tunnel syndrome which was confirmed using electromyography.

Comprehensive genetic testing was done at 8 years of age. A chromosomal microarray (Illumina CytoSNP-850v1.1) was performed and excluded any pathogenic copy number variants. Subsequently, whole exome sequencing was performed at Cincinnati Children's Hospital on the patient and her parents using previously described methods [11]. As neither parent was affected, both recessive and de novo dominant inheritance models were investigated. The patient was found to have a de novo heterozygous mutation in FBN1 gene p.Tyr1696Asp (Figure $1(\mathrm{~g})$ ). This variant has not previously been reported in the UMD-FBN1 mutation database (www.umd.be/FBN1/) and is not present in a large healthy control database (gnomad.broadinstitute.org), but it is predicted to be damaging by UMD-predictor, CADD and MutationTaster2 [12-14].

After genetic testing, a trial with high-dose $\mathrm{rGH}$ treatment at a dose $0.06 \mathrm{mg} / \mathrm{kg} /$ day was started due to extremely severe dwarfism which resulted in incremental growth of 1.5 $\mathrm{cm}$ per 6 months of treatment (Figure $1(\mathrm{e})$ ) with a subsequent increase of IGF-1 level $(318.8 \mathrm{ng} / \mathrm{ml}$, between the 50 th and 90th percentile) after 3 months of treatment and $151 \mathrm{ng} / \mathrm{ml}$ (between the 10th and 50th percentile) after 6 months of treatment accordingly.

Patient 2 (P2) is a Ukrainian boy who was born as the first child of nonconsanguineous white parents via spontaneous term delivery. He was born at a gestational age of 40 weeks with a birth weight of $4050 \mathrm{~g}\left(85^{\text {th }}-97^{\text {th }}\right.$ percentile $)$ and birth length of $55 \mathrm{~cm}$ ( $>97^{\text {th }}$ percentile). Familial stature was quite tall with a maternal height of $180 \mathrm{~cm}(+2.9 \mathrm{SD})$ and paternal height of $195 \mathrm{~cm}$ (+3 SD) (Figure 2(a)). Progressive postnatal growth retardation developed beginning at 1 year of age. Prior to 2 years of age, the child had frequent respiratory infections. At the age of 2 years, a tracheostomy was placed due to acute edema of the throat, asphyxia, and pneumonia. In Ukraine, all further attempts to remove the tracheostomy with dilation and resection of pathologic tissue were unsuccessful.

The patient had many dysmorphic features and other physical abnormalities including an indented nasal bridge, elongation of the eye lashes, prominent upper jaw, peripheral edema, thick lips, tapered fingers, stiff interphalangeal joints, and short hands and fingers. At 2 years of age, comprehensive screening for metabolic disorders was completed including mannosidosis, fucosidosis, metachromatic leukodystrophy, Sandhoff disease, lysosomal storage diseases, GM1 gangliosidosis, Krabbe disease, and mucopolysaccharidosis (types 13 and 6). However, all metabolic testing was within normal limits. Thereafter, the child was referred to two European clinics in Germany and Denmark with the aim of removing the tracheostomy, but in both cases video laryngoscopy showed large adenoid-like tissue within the rhinopharynx and oropharynx which could cause persistent obstruction. 


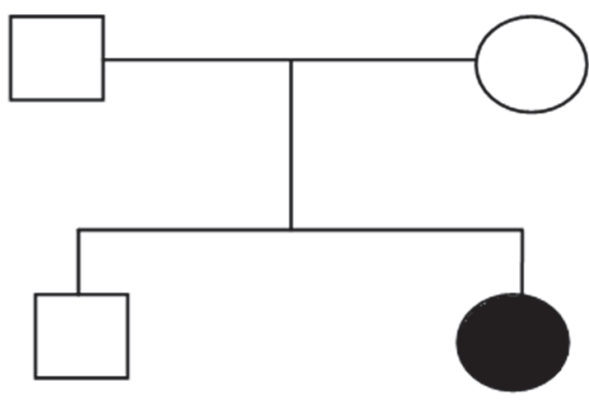

(a) Pedigree of P1 with her unaffected father, mother, and brother

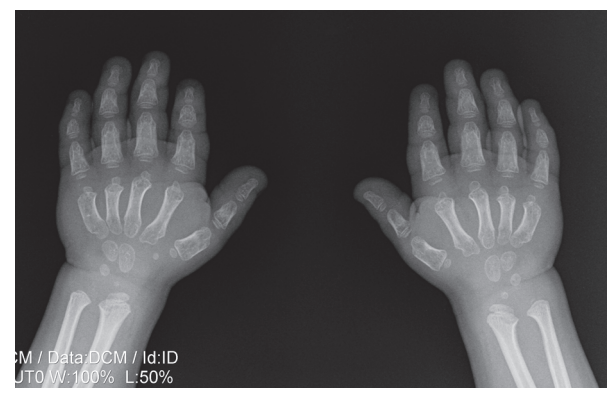

(c) Bone age in P1

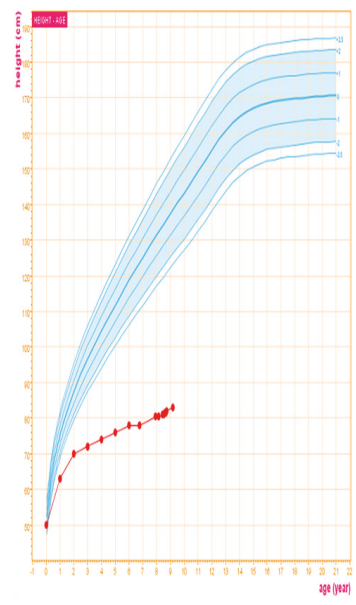

(e) Growth chart of P1

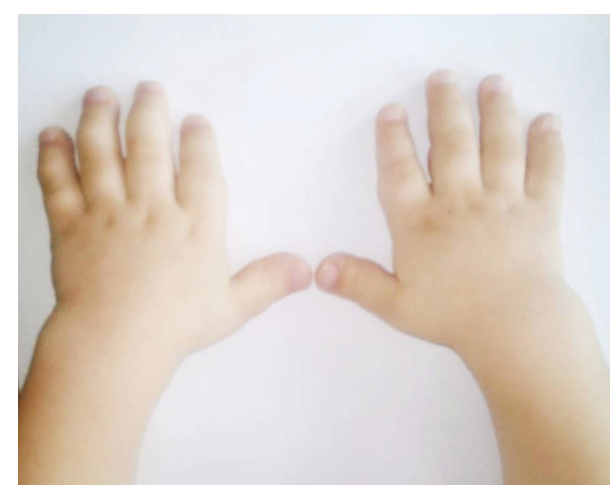

(b) Hands of the P1

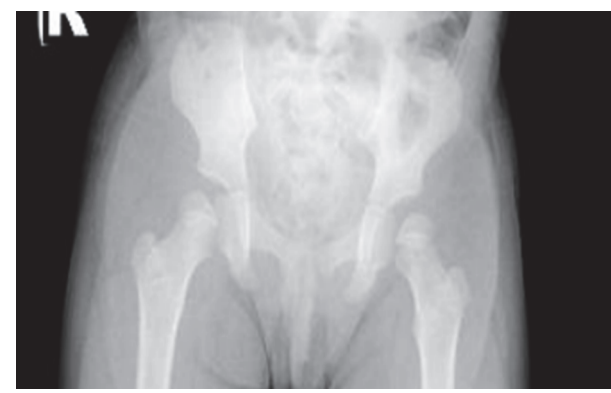

(d) Skeletal X-ray of the hips in P1

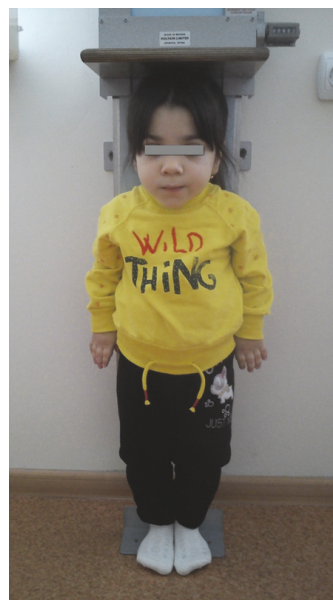

(f) Photograph at the age of 9 years and 3 months
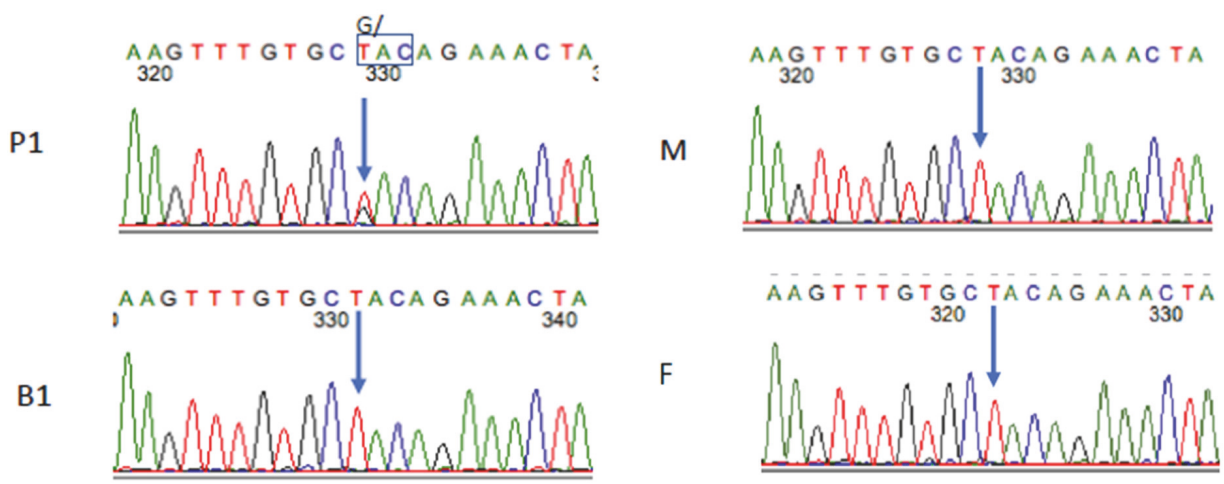

(g) Heterozygous FBN1 mutation c.5086T>G (p.Tyr1696Asp) in exon 42 in P1, brother (B1), mother (M), and father $(\mathrm{F})$ 


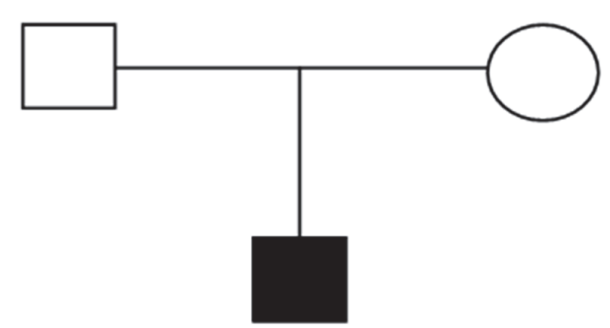

(a) Pedigree of P2 with his unaffected father and mother

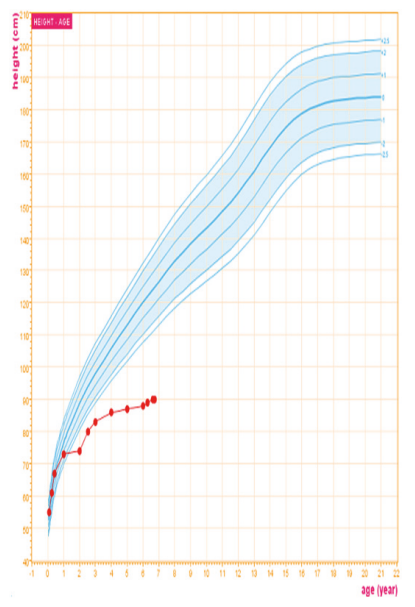

(c) Growth chart in P2

P2

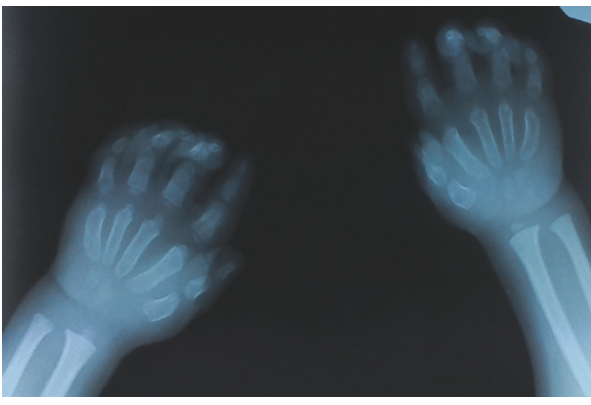

(d) Bone age in P2

(b) Photograph at the age of 4 years

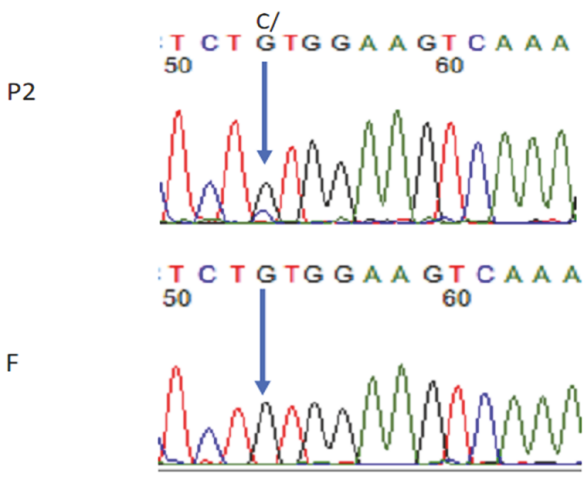

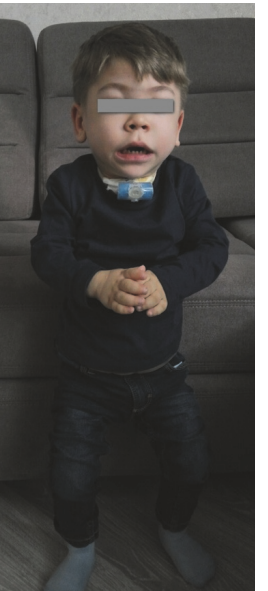

(e) Heterozygous FBN1 mutation c.5243G >C (p.Cys1748Ser) in exon 43 in P2, mother (M), and father (F)

Figure 2

Therefore, it was recommended that the tracheostomy remain in place permanently. Due to the suspicion of mucopolysaccharidosis or mucolipidosis, a targeted next generation sequencing panel of 99 genes was performed; however no variants were identified.

The patient was first examined by an endocrinologist at 4 years of age (Figure 2(b)). Blood samples showed normal hematology, chemistry, and thyroid hormone function. IGF1 was $41.8 \mathrm{ng} / \mathrm{ml}(<5$ th percentile); however clonidinestimulated GH peak was normal (12.8 ng/ml). IGF-1 generation test showed a good response to stimulation (after three days of GH administration at a dose of $0.03 \mathrm{mg} / \mathrm{kg} / \mathrm{d}$ IGF-1 was $205.5 \mathrm{ng} / \mathrm{ml}$ ). However, treatment with $\mathrm{rGH}$ at a dose 0.03 $\mathrm{mg} / \mathrm{kg} /$ day for 5 months did not result in catch-up growth $(0$ 
cm per 5 months of treatment) (Figure 2(c)). He had a normal male karyotype (46 XY). His bone age was 2 years (assessed by the Greulich and Pyle method) with noticeable cone-shaped epiphyses (Figure 2(d)). Echocardiography was normal.

Similar to the first patient, comprehensive genetic testing was done at 6 years of age. Chromosomal microarray was negative but whole exome sequencing identified a de novo heterozygous mutation in FBN1 p.Cys1748Ser (Figure 2(e)). Similar to the variant found in the first patient, this mutation was not previously described in the UMD-FBN1 database and was not present in the gnom $A D$ healthy control database, but it is predicted as pathogenic by UMD-predictor, CADD and MutationTaster2 [12-14].

After genetic testing, a trial with high-dose rGH treatment at a dose of $0.06 \mathrm{mg} / \mathrm{kg} /$ day was started and showed no improvement in growth $(0 \mathrm{~cm}$ per 3 months of treatment, Figure 2(c)).

Treatment with anti-inflammatory drugs for his airway issues was started including azithromycin and inhaled or nebulized mometasone.

\section{Discussion}

The Ukrainian Pediatric Growth Hormone Registry was created in 2012 to include children diagnosed with short stature identified by regional Ukrainian pediatric endocrinologists. The number of cases with hypopituitarism in Ukraine in 2016 was 962 (a prevalence of 1 in 7914 for the pediatric population in 2016), and there were an additional 315 cases of Turner syndrome (1:24171). The registry includes also additional patients with syndromic dwarfism who are not receiving $\mathrm{GH}$ treatment. However due to the lack of access to genetic diagnostics in Ukraine, a case of GD has not been previously described.

The diagnosis of GD can be based on clinical findings including proportionate short stature, very short hands and feet, progressive joint limitation and contractures, distinctive facial features (round, full face; small nose with anteverted nostrils; broad nasal bridge; thin upper lip with flat philtrum), thickened skin, and progressive cardiac valvular disease [14]. Additional features include recurrent respiratory and middle-ear infections, tracheal stenosis, and hepatomegaly $[1,6]$.

In this report, we describe two patients with molecular defects in FBN1 leading to severe short stature which was resistant to $\mathrm{GH}$ treatment. Both patients remained undiagnosed for many years until exome sequencing confirmed the etiology of their clinical presentations. Both children had facial dysmorphisms (broad nasal bridge, a bulbous nose, elongation of the eye lashes, small hands and fingers, progressive joint limitation, and contractures). However one of the patients (P1) had progressive cardiac pathology which was found at the age of 7 years, and the second patient had severe pathology of the respiratory system with tracheal stenosis requiring tracheostomy placement at 2 years of age. In patients with $\mathrm{GD}$, rapid progression of cardiac pathology has been also described $[15,16]$ leading to the necessity of timely and adequate cardiac health supervision including the use of valve replacement when indicated. Severe respiratory problems were described as a leading cause of early death in patients with GD $[6,7]$ and often require tracheostomy placement.

Radiographic findings usually include delayed bone age, broad proximal phalanges, cone-shaped epiphyses, ovoid vertebral bodies, shortened tubular bones of the hands and feet, and small capital femoral epiphyses $[1,4,17]$. Skeletal survey in both our patients showed cone-shaped epiphyses and shortening of tubular bones. One of the patients (P1) had dysplasia of the hip and Madelung deformity with carpal tunnel syndrome requiring a carpal tunnel release. Neither patient had hepatomegaly which was confirmed by abdominal ultrasound.

Genetic testing confirmed two newly identified mutations in FBN1 (p.Tyr1696Asp and p.Cys1748Ser) which are located in the TB5 domain. Other mutations in this FBN1 domain are linked with Marfan syndrome and WMS. Sanger sequencing confirmed the presence of the de novo variants in each of the patients. The variants were absent in the parents. Although the two reported mutations are new, a few mutations have been described in the same amino acids: p.Tyr1696Cys (X2) [6], p.Cys1748Phe [18], and p.Cys1748Arg [19]. In comparing the phenotypes of patients with the similar variants, it is noteworthy that a patient with p.Tyr1696Cys also required tracheostomy placement at 3 years of age. That patient additionally had mitral stenosis and insufficiency and died at 9 years of age [6]. The patients with p.Cys1748Phe and p.Cys1748Arg had WMS phenotype with ectopia lentis and in the case of the Cys1748Arg mutation an acute thoracic aortic dissection at 38 years of age $[18,19]$.

There is insufficient data regarding $\mathrm{GH}$ treatment in patients with various skeletal dysplasias [20] especially in those with acromelic dysplasia syndromes. However, there have been a variety of reports with conflicting results $[21,22]$ presumably due to the underlying genetic heterogeneity and different treatment regimens. A trial of recombinant IGF-1 therapy (mecasermin) in a single patient was also previously described [4]. As GD patients are characterized by severe short stature (>3 SD) [6], long-term follow-up is needed to study the advisability of $\mathrm{GH}$ treatment regimens in an effort to improve their final height.

Taking into account the severity of dwarfism in our patients ( $>-6$ and $-8 \mathrm{SD}$ ) with resistance to $\mathrm{rGH}$ treatment and severe multiorgan involvement (rapid progression of cardiac pathology and tracheostomy placement), there is a need for further research into alternate treatment modalities. Annual multidisciplinary examination is recommended to provide a comprehensive evaluation of all involved organ systems in order to assess for known comorbidities of GD.

\section{Abbreviations}

AD: Acromicric dysplasia

GD: Geleophysic dysplasia

$\mathrm{GH}$ : Growth hormone

rGH: Recombinant GH

WMS: Weill-Marchesani syndrome. 


\section{Ethical Approval}

All the procedures performed in the studies involving patients were in accordance with the ethical standards of the institution on clinical practice and with the 1964 Helsinki Declaration, as amended.

\section{Consent}

The parents or legal guardians of patients signed informedconsent forms in which they agreed to the treatment and all the diagnostic procedures required.

\section{Disclosure}

Ipsen contributed to the publication costs.

\section{Conflicts of Interest}

The authors declare that they have no conflicts of interest.

\section{Authors' Contributions}

Evgenia Globa was responsible for conception and design of the study, data acquisition, preparation of the manuscript, finding relevant references, and final approval of the manuscript. Nataliya Zelinska designed the analyses; contributed to discussion; and wrote, reviewed, and edited the manuscript. Andrew Dauber performed and interpreted genetic testing; conceptualized and designed the study; and critically reviewed and revised the manuscript. Andrew Dauber is the guarantor and approved the final manuscript as submitted.

\section{Acknowledgments}

The authors are grateful to Professor Henrik Thybo Christesen and Christian Godbale, Hans Christian Andersen Children's Hospital, Odense University Hospital, Odense, Denmark. Henrik Thybo Christesen helped with admission of P2 and investigation. Christian Godbale performed a video laryngoscopy. Special thanks are due to Professor Mike Rutter, Cincinnati Children's Hospital Medical Center, who helped with further clinical management of P2. Shcherbak Yuliya, M.D., Ph.D., (National Hospital "OHMATDYT", Kyiv, Ukraine) helped with the local genetic investigation.

\section{References}

[1] C. Le Goff and V. Cormier-Daire, "Genetic and molecular aspects of acromelic dysplasia," Pediatric Endocrinology Reviews, vol. 6, pp. 418-423, 2009.

[2] B. F. Pontz, H. Stoss, F. Henschke, P. Freisinger, A. Karbowski, and J. Spranger, "Clinical and ultrastructural findings in three patients with geleophysic dysplasia," American Journal of Medical Genetics, vol. 63, no. 1, pp. 50-54, 1996.

[3] C. Le Goff and V. Cormier-Daire, "From tall to short: The role of TGF $\beta$ Signaling in growth and its disorders," American Journal of Medical Genetics Part C: Seminars in Medical Genetics, vol. 160, no. 3, pp. 145-153, 2012.

[4] C. de Bruin, C. Finlayson, M. F. A. Funari et al., "Two Patients with Severe Short Stature due to a FBN1 Mutation (p.Ala1728Val) with a Mild Form of Acromicric Dysplasia," Hormone Research in Paediatrics, vol. 86, no. 5, pp. 342-348, 2016.

[5] C. Le Goff, F. Morice-Picard, N. Dagoneau, L. W. Wang, C. Perrot et al., "ADAMTSL2 mutations in geleophysic dysplasia demonstrate a role for ADAMTS-like proteins in TGF-beta bioavailability regulation," Nature Genetics, vol. 40, no. 9, pp. 1119-1123, 2008.

[6] C. Le Goff, C. Mahaut, L. Wang, S. Allali, A. Abhyankar et al., "Mutations in the TGFb Binding-Protein-Like Domain 5 of FBN1 Are Responsible for Acromicric and Geleophysic Dysplasias," The American Journal of Human Genetics, vol. 89, pp. 7-14, 2011.

[7] A. M. McInerney-Leo, C. Le Goff, P. J. Leo et al., "Mutations in LTBP3 cause acromicric dysplasia and geleophysic dysplasia," Journal of Medical Genetics, vol. 53, no. 7, pp. 457-464, 2016.

[8] M. Aubart, L. Benarroch, P. Arnaud, G. Collod-Béroud, G. Jondeau, and C. Boileau, Molecular Genetics of the Fibrillinopathies, Wiley Online Library, 2016, https://doi.org/10.1002/ 9780470015902.a0025314.

[9] L. Y. Sakai, D. R. Keene, M. Renard, and J. De Backer, "FBN1: The disease-causing gene for Marfan syndrome and other genetic disorders," Gene, vol. 592, no. 1, pp. 279-291, 2016.

[10] H. C. Dietz, G. R. Cutting, R. E. Pyeritz et al., "Marfan syndrome caused by a recurrent de novo missense mutation in the fibrillin gene," Nature, vol. 352, no. 6333, pp. 337-339, 1991.

[11] C. de Bruin, V. Mericq, S. F. Andrew et al., "An XRCC4 splice mutation associated with severe short stature, gonadal failure, and early-onset metabolic syndrome," The Journal of Clinical Endocrinology \& Metabolism, vol. 100, no. 5, pp. E789-E798, 2015.

[12] D. Salgado, J.-P. Desvignes, G. Rai et al., "UMD-Predictor: A High-Throughput Sequencing Compliant System for Pathogenicity Prediction of any Human cDNA Substitution," Human Mutation, vol. 37, no. 5, pp. 439-446, 2016.

[13] M. Kircher, D. M. Witten, P. Jain, B. J. O’Roak, G. M. Cooper, and J. Shendure, "A general framework for estimating the relative pathogenicity of human genetic variants," Nature Genetics, vol. 46, no. 3, pp. 310-315, 2014.

[14] J. M. Schwarz, D. N. Cooper, M. Schuelke, and D. Seelow, "MutationTaster2: mutation prediction for the deep-sequencing age," Nature Methods, vol. 11, no. 4, pp. 361-362, 2014.

[15] G. Rama, W. K. Chung, C. M. Cunniff, and U. Krishnan, "Rapidly progressive mitral valve stenosis in patients with acromelic dysplasia," Cardiology in the Young, vol. 27, no. 4, pp. 797-800, 2017.

[16] A. Scott, S. Yeung, D. F. Dickinson, G. Karbani, and Y. J. Crow, "Natural history of cardiac involvement in geleophysic dysplasia," American Journal of Medical Genetics, vol. 132, no. 3, pp. 320-323, 2005.

[17] K. Hasegawa, C. Numakura, H. Tanaka et al., "Three cases of Japanese acromicric/geleophysic dysplasia with FBN1 mutations: A comparison of clinical and radiological features," Journal of Pediatric Endocrinology and Metabolism, vol. 30, no. 1, pp. 117-121, 2017.

[18] Y. Wang, H. Zhang, J. Ye, L. Han, and X. Gu, “Three novel mutations of the FBN1 gene in Chinese children with acromelic 
dysplasia," Journal of Human Genetics, vol. 59, no. 10, pp. 563567, 2014.

[19] A. Cecchi, N. Ogawa, H. R. Martinez et al., "Missense mutations in FBN1 exons 41 and 42 cause Weill-Marchesani syndrome with thoracic aortic disease and Marfan syndrome," American Journal of Medical Genetics Part A, vol. 161, no. 9, pp. 2305-2310, 2013.

[20] L. Hagenäs and T. Hertel, "Skeletal dysplasia, growth hormone treatment and body proportion: Comparison with other syndromic and non-syndromic short children," Hormone Research, vol. 60, no. 3, pp. 65-70, 2003.

[21] H. S. Jin, H. Y. Song, S. Y. Cho et al., "Acromicric dysplasia caused by a novel heterozygous mutation of FBN1 and effects of growth hormone treatment," Annals of Laboratory Medicine, vol. 37, no. 1, pp. 92-94, 2017.

[22] L. Faivre, M. Le Merrer, C. Baumann et al., "Acromicric dysplasia: Long term outcome and evidence of autosomal dominant inheritance," Journal of Medical Genetics, vol. 38, no. 11, pp. 745749, 2001. 


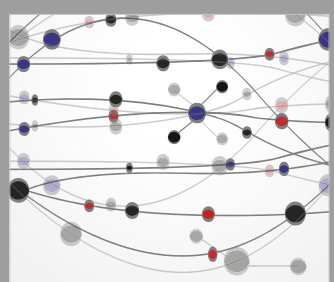

The Scientific World Journal
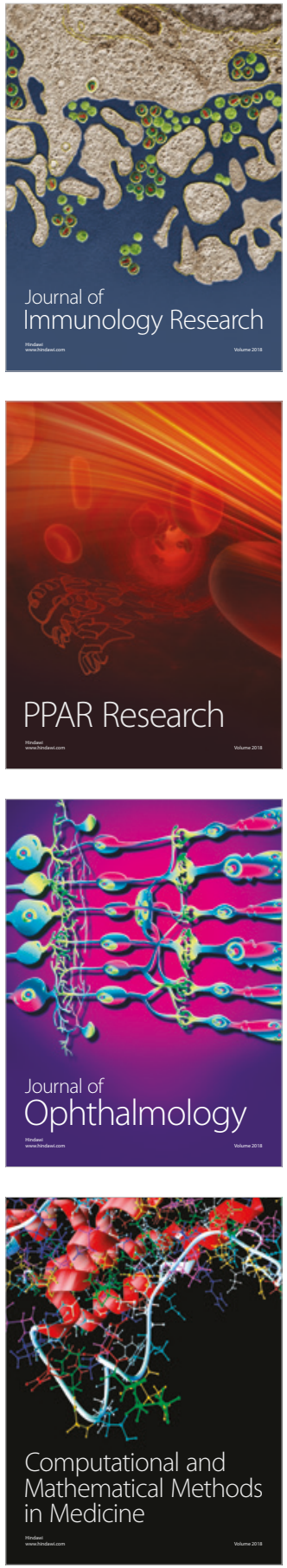

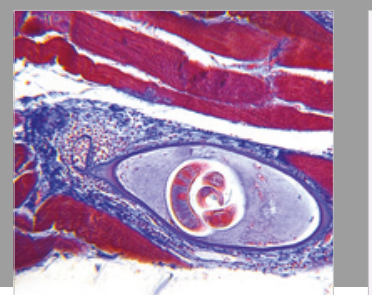

Gastroenterology Research and Practice

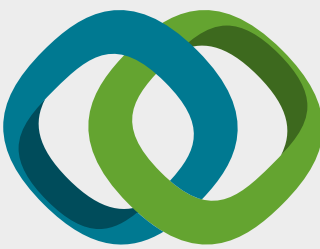

\section{Hindawi}

Submit your manuscripts at

www.hindawi.com
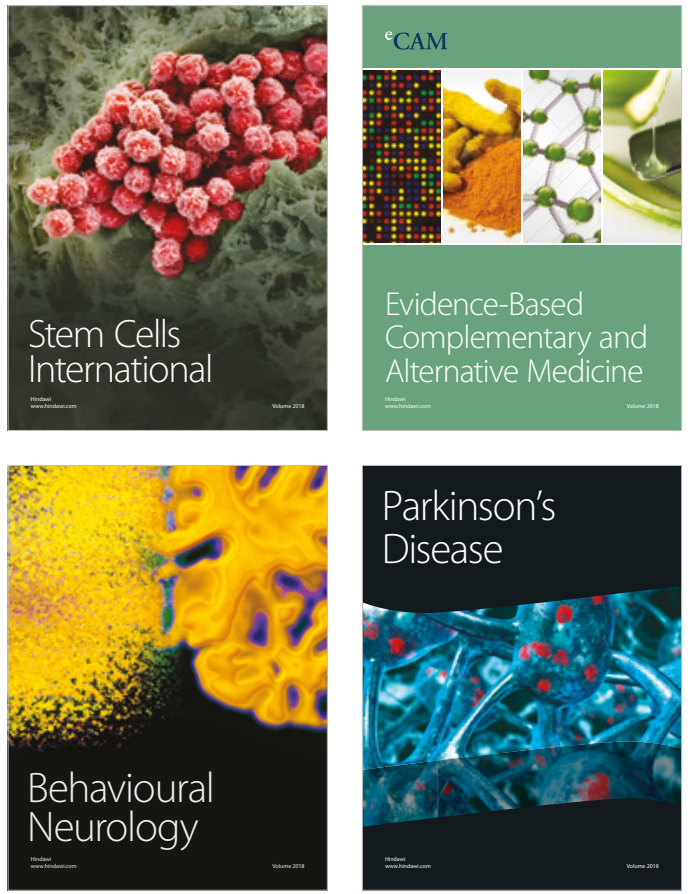

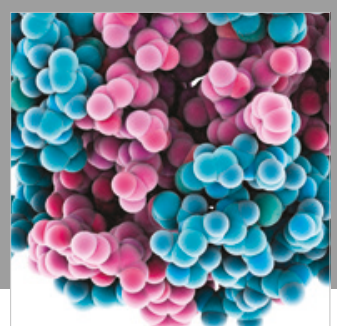

ournal of

Diabetes Research

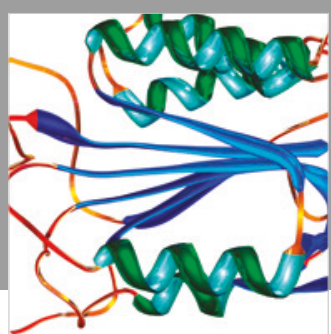

Disease Markers
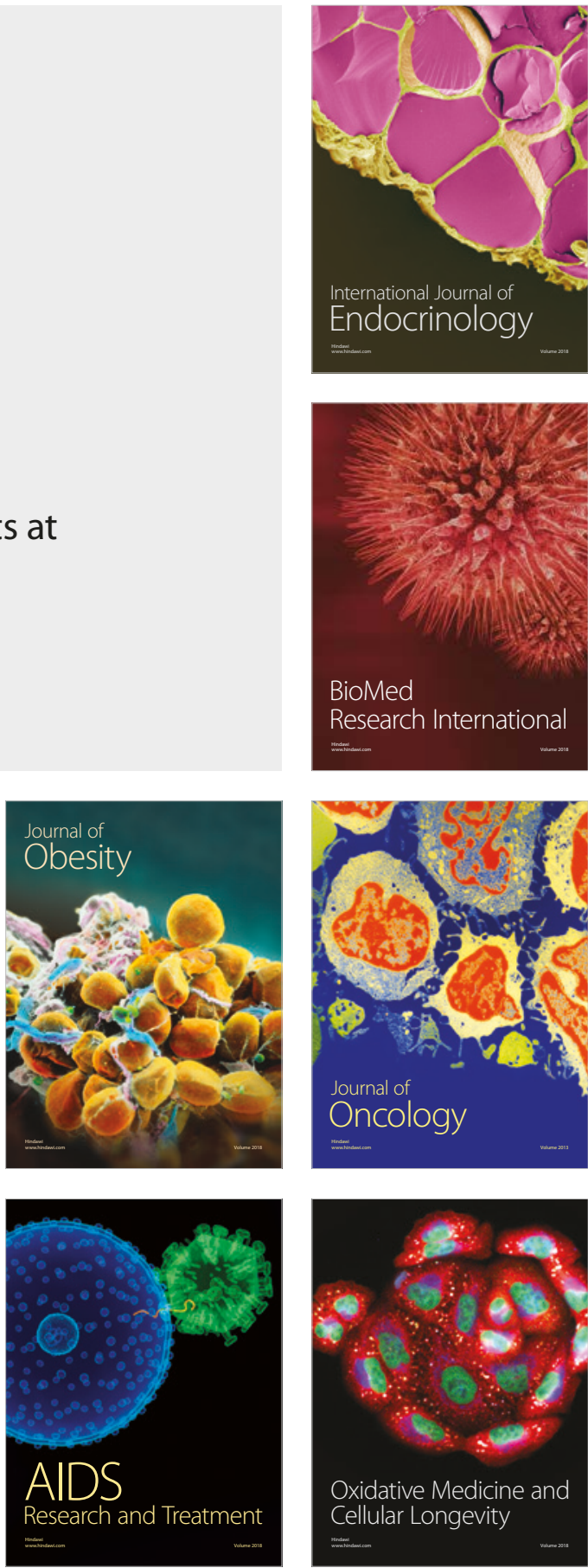